FUNCIONES DE VOLUMEN Y FACTOR DE FORMA PARA Pseudotsuga menziesii (PINO OREGON). Hans Grosse Werner. Dr. Ingeniero Forestal, División Regional, Instituto Forestal. Barros Arana 121. Concepción - Chile.

\title{
INTRODUCCION
}

Con el objeto de entregar antecedentes básicos para la planificación forestal de los productos de Pseudotsuga menziesii, se realizaron estudios orientados a entregar el máximo de información sobre las variables de estado de la especie y su crecimiento (GROSSE y KANNEGIESSER 1988). Dentro de este marco de acción, se construyeron tablas de volumen total por árbol y por troza. Los resultados de esta parte del estudio se presentan a continuación.

El sector donde se realizó el muestreo corresponde al fundo Voipir ubicado a $6 \mathrm{Km}$ al sur del pueblo de Villarrica en los $72^{\circ} 18^{\prime}$ Long. O. y $39^{\circ} 21^{\prime}$ Lat. S. Los bosques se ubican sobre lomajes suaves que están entre los 300 y 750 m.s.n.m.

\section{ANTECEDENTES METODOLOGICOS}

\section{El muestreo en terreno}

Para cumplir con los objetivos planteados se realizó un muestreo que abarcó distintas clases de edad. Una descripción de los sectores de donde se extrajeron las muestras se entrega en la Tabla 1.

TABLA 1

VARIABLES DE ESTADO DE LOS RODALES MUESTREADOS

\begin{tabular}{|c|c|c|c|c|}
\hline Variables de estado & A & B & C & D \\
\hline Número de árboles por ha & 940 & 780 & 480 & 350 \\
\hline DAP medio cuadr. $(\mathrm{cm})$ & 22,7 & 23,1 & 37,0 & 40,1 \\
\hline Edad de! rodal (años) & $15 \cdot 16$ & $17-18$ & $26 \cdot 32$ & $33-35$ \\
\hline Area basal $\left(\mathrm{m}^{2}\right)$ & 38,0 & 32,6 & 51,7 & 44,2 \\
\hline Altura Total (m) & 16,4 & 16,3 & 29,6 & 31,5 \\
\hline Base muestreal & 10 & 4 & 7 & 7 \\
\hline
\end{tabular}

Los individuos provenientes de rodales de aproximadamente 14 años de edad fueron cortados prácticamente a nivel del suelo y seccionados en trozas cortas de tamaño variable. Para los árboles de rodales mayores se dejó un tocón de aproximadamente $25 \mathrm{~cm}$.

Los árboles sobre $25 \mathrm{~cm}$ de DAP se seccionaron en trozas de $3,35 \mathrm{~m}$, correspondiendo este largo al standard utilizado en las ventas de la empresa dueña del fundo Voipir. La muestra total extraida corresponde a 28 árboles. Su distribución por clase diamétrica se presenta en la Tabla 2 
TABLA 2

DISTRIBUCION DE LA MUESTRA POR CLASE DAP

\begin{tabular}{|c|c|c|}
\hline \multirow[b]{2}{*}{$\begin{array}{l}\text { Clase DAP } \\
\text { (cm) }\end{array}$} & \multicolumn{2}{|c|}{ NUMERO DE MUESTRAS PARA } \\
\hline & $\begin{array}{c}\text { Tabla de Vol. } \\
\text { Total }\end{array}$ & $\begin{array}{c}\text { Tabla de Vol. } \\
\text { por troza }(3,35 \mathrm{~m})\end{array}$ \\
\hline $5,0-9,9$ & 1 & - \\
\hline $10,0 \cdot 14,9$ & 2 & - \\
\hline $15,0-19,9$ & 4 & - \\
\hline $20,0-24,9$ & 5 & - \\
\hline $25,0-29,9$ & 4 & 3 \\
\hline $30,0-34,9$ & 3 & 3 \\
\hline $35,0 \cdot 39,9$ & 4 & 4 \\
\hline $40,0-44,9$ & 2 & 2 \\
\hline $45,0 \cdot 49,9$ & 2 & 2 \\
\hline $50,0-54,9$ & 1 & 1 \\
\hline Total & 28 & 15 \\
\hline
\end{tabular}

\section{La estimación de los volúmenes}

Se construyeron tablas de volumen total y por troza.

Los modelos probados para la construcción de tablas de volumen total son:
(a) $\mathrm{V}=\mathrm{b}_{0}+\mathrm{b}_{1} \mathrm{D}^{2} \mathrm{H}$
(b) $\mathrm{V}=\mathrm{b}_{0}+\mathrm{b}_{2} \mathrm{D}^{3}$
(c) $\mathrm{V}=\mathrm{b}_{0}+\mathrm{b}_{1} \mathrm{H}+\mathrm{b}_{2} \mathrm{D}^{2} \mathrm{H}$

Donde:

V : Volumen total en $\mathrm{m}^{3} \mathrm{ssc}$
D : DAP con corteza en $\mathrm{cm}$

Los modelos probados para la construcción de tablas de volumen por troza son:
(a) $\mathrm{V}=\mathrm{b}_{0}+\mathrm{b}_{1} \mathrm{D}$
(b) $\mathrm{V}=\mathrm{b}_{0}+\mathrm{b}_{1} \mathrm{D}^{2}$
(c) $\mathrm{V}=\mathrm{b}_{0}+\mathrm{b}_{1} \mathrm{D}+\mathrm{b}_{2} \mathrm{D}^{2}$

Donde:

$\mathrm{V}$ : Volumen s.s.c. por troza de $3,35 \mathrm{~m}$ de largo en $\mathrm{m}^{3}$

D : DAP con corteza en $\mathrm{cm}$ 
Para el cálculo del volumen por troza se empleó la fórmula de Smalian (HUSCH, MILLER y BEERS, 1982), considerando trozas hasta un diámetro límite sin corteza de $10 \mathrm{~cm}$.

La bondad de ajuste de los modelos se presenta con la correlación y el error standard.

\section{RESULTADOS}

A continuación se presentan las funciones para estimar el volumen total, por troza y el factor de forma para Pseudotsuga menziesii.

\section{Volumen total}

Tabla 3 .

Las funciones para estimar el volumen total para Pseudotsuga menziesii se presentan en la

TABLA 3

COEFICIENTES Y BONDAD DE AJUSTE PARA TRES MODELOS DE VOLUMEN TOTAL EN FUNCION DEL DAP Y LA ALTURA TOTAL PARA PSEUDOTSUGA MENZIESII (Fundo Voipir - Villarrica)

\begin{tabular}{|ccccccc|}
\hline Modelos & $\mathbf{b}_{\mathbf{0}}$ & $\mathbf{b}_{\mathbf{1}}$ & $\mathbf{b}_{\mathbf{2}}$ & $\begin{array}{c}\text { Coef. Corr. } \\
\mathbf{( r )}\end{array}$ & $\begin{array}{c}\text { Error Std. } \\
\mathbf{S}_{\mathbf{y}} \cdot \mathbf{x}\end{array}$ & $\begin{array}{c}\mathbf{N}^{\circ} \text { Observ. } \\
\mathbf{n}\end{array}$ \\
\hline & & & & & & \\
$\mathrm{a}$ & 0,030764 & 0,000028 & --- & 0,99 & 0,083 & 28 \\
$\mathrm{~b}$ & 0,084547 & 0,000019 & -- & 0,99 & 0,085 & 28 \\
$\mathrm{c}$ & $-0,004390$ & 0,002481 & 0,000027 & 0,99 & 0,084 & 28 \\
\hline
\end{tabular}

Modelos
(a) $\mathrm{V}=\mathrm{b}_{0}+\mathrm{b}_{1} \mathrm{D}^{2} \mathrm{H}$
(b) $\mathrm{V}=\mathrm{b}_{0}+\mathrm{b}_{1} \mathrm{D}^{3}$
(c) $\mathrm{V}=\mathrm{b}_{0}+\mathrm{b}_{1} \mathrm{H}+\mathrm{b}_{2} \mathrm{D}^{2} \mathrm{H}$

Donde:

$\mathrm{V}$ : Volumen total en $\mathrm{m}^{3} \mathrm{ssc}$

D : DAP con corteza en $\mathrm{cm}$

$b_{1}, b_{0}$ : Coeficientes de regresión y 4).

Para construir la tabla de volumen total para Pseudotsuga se eligió el modelo a (Tablas 3 
TABLA 4

VOLUMEN CUBICO $\left(\mathrm{m}^{3}\right.$ s.S.c.) POR CLASE DAP Y ALTURA TOTAL PARA PSEUDOTSUGA MENZIESII

(Fundo Voipir - Villarrica)

\begin{tabular}{|c|c|c|c|c|c|c|}
\hline \multirow{3}{*}{$\begin{array}{l}\text { CLASE } \\
\text { DAP } \\
\text { (cm) }\end{array}$} & \multicolumn{6}{|c|}{ CLASE ALTURA (m) } \\
\hline & 10 & 15 & 20 & 25 & 30 & 35 \\
\hline & \multicolumn{6}{|c|}{ Volumen cúbico $\left(\mathrm{m}^{3}\right.$ s.s.c. $)$} \\
\hline 10 & 0,0587 & 0,0727 & & & & \\
\hline 15 & 0,0937 & 0,1252 & 0,1567 & & & \\
\hline 20 & 0,1427 & 0,1987 & 0,2547 & & & \\
\hline 25 & & 0,2932 & 0,3807 & 0,4682 & & \\
\hline 30 & & 0,4087 & 0,5347 & 0,6607 & 0,7867 & \\
\hline 35 & & & 0,7167 & 0,8882 & 1,0597 & 1,2312 \\
\hline 40 & & & & 1,1507 & 1,3747 & 1,5987 \\
\hline 45 & & & & & 1,7317 & 2,0152 \\
\hline 50 & & & & & 2,1307 & 2,4807 \\
\hline 55 & & & & & 2,5717 & 2,9952 \\
\hline
\end{tabular}

(a) $\mathrm{V}=0,030764+0,000028 \mathrm{D}^{2} \mathrm{H}$

-.-.: El rango muestreal se enmarcó entre líneas discontinuas. 


\section{Volumen por troza}

Tabla 5 .

Los coeficientes e indicadores de ajuste para los modelos probados se presentan en la

TABLA 5

COEFICIENTES Y BONDAD DE AJUSTE POR TROZA DE 3,35 m DE LARGO CON UN DIAMETRO LIMITE DE UTILIZACION DE $10 \mathrm{~cm}$ PARA PSEUDOTSUGA MENZIESII

(Fundo Voipir - Villarrica)

\begin{tabular}{|c|c|c|c|c|c|c|}
\hline Troza & b。 & $b_{1}$ & $b_{2}$ & $\begin{array}{c}\text { Error Standard } \\
\qquad \mathbf{S} \cdot \mathbf{x}\end{array}$ & $\begin{array}{c}\text { Coef. Correl. } \\
\mathbf{r}\end{array}$ & $\begin{array}{c}\mathrm{N}^{\circ} \text { de } \\
\text { muestra por } \\
\text { clase de troza } \\
\mathrm{n}\end{array}$ \\
\hline \multicolumn{7}{|c|}{ MODELO (a) } \\
\hline $\begin{array}{l}1 \\
2 \\
3 \\
4 \\
5 \\
6 \\
7\end{array}$ & $\begin{array}{l}-0,240370 \\
-0,180440 \\
-0,176610 \\
-0,115240 \\
-0,085630 \\
-0,012390 \\
-0,002717\end{array}$ & $\begin{array}{l}0,015465 \\
0,011266 \\
0,010109 \\
0,007398 \\
0,005578 \\
0,002773 \\
0,001730\end{array}$ & & $\begin{array}{l}0,033 \\
0,022 \\
0,021 \\
0,015 \\
0,021 \\
9,025 \\
0,012\end{array}$ & $\begin{array}{l}0,97 \\
0,98 \\
0,96 \\
0,97 \\
0,91 \\
0,66 \\
0,49\end{array}$ & $\begin{array}{r}15 \\
15 \\
14 \\
14 \\
13 \\
10 \\
5\end{array}$ \\
\hline $\begin{array}{l}1 \\
2 \\
3 \\
4 \\
5 \\
6 \\
7\end{array}$ & $\begin{array}{l}0,058689 \\
0,023008 \\
0,020690 \\
0,031518 \\
0,026549 \\
0,048306 \\
0,038364\end{array}$ & $\begin{array}{l}\text { MOD } \\
0,000191 \\
0,000148 \\
0,000124 \\
0,000089 \\
0,000066 \\
0,000030 \\
0,000020\end{array}$ & $O$ (b) & $\begin{array}{l}0,034 \\
0,017 \\
0,019 \\
0,017 \\
0,024 \\
0,027 \\
0,012\end{array}$ & $\begin{array}{l}0,96 \\
0,99 \\
0,97 \\
0,96 \\
0,88 \\
0,61 \\
0,50\end{array}$ & $\begin{array}{r}15 \\
15 \\
14 \\
14 \\
13 \\
10 \\
5\end{array}$ \\
\hline \multicolumn{7}{|c|}{ *MODELO (c) } \\
\hline $\begin{array}{l}1 \\
2 \\
3 \\
4 \\
5 \\
6 \\
7\end{array}$ & $\begin{array}{r}-0,180830 \\
0,001388 \\
0,097933 \\
-0,190180 \\
-0,367050 \\
-0,430340 \\
-0,367170\end{array}$ & $\begin{array}{r}0,012362 \\
0,001174 \\
-0,003910 \\
0,011225 \\
0,019997 \\
0,023363 \\
0,019863\end{array}$ & $\begin{array}{r}0,000038 \\
0,000133 \\
0,000172 \\
-0,000040 \\
-0,000170 \\
-0,000240 \\
-0,000220\end{array}$ & $\begin{array}{l}0,034 \\
0,018 \\
0,020 \\
0,015 \\
0,019 \\
0,021 \\
0,015\end{array}$ & $\begin{array}{l}0,97 \\
0,99 \\
0,97 \\
0,97 \\
0,94 \\
0,81 \\
0,52\end{array}$ & $\begin{array}{r}15 \\
15 \\
14 \\
14 \\
13 \\
10 \\
5\end{array}$ \\
\hline
\end{tabular}

(a) $\mathrm{V}=\mathrm{b}_{0}+\mathrm{b}_{1} \mathrm{D}$

(b) $\mathrm{V}=\mathrm{b}_{0}+\mathrm{b}_{1} \mathrm{D}^{2}$

(c) $\quad V=b_{0}+b_{1} D+b_{2} D^{2}$

$\mathrm{V}=$ Volumen $\left(\mathrm{m}^{3}\right.$ s.s.c. $)$

$\mathrm{D}=\operatorname{DAP}(\mathrm{cm})$

* : Modelo elegido 
El volumen por troza para Pseudotsuga menziesii se presenta en la Tabla 6.

TABLA 6

VOLUMEN POR TROZA PARA PSEUDOTSUGA MENZIESII

(Fundo Voipir - Villarrica)

\begin{tabular}{|c|c|c|c|c|c|c|c|}
\hline \multirow{2}{*}{$\begin{array}{l}\text { CLASE } \\
\text { DAP } \\
(\mathrm{cm})\end{array}$} & \multicolumn{6}{|c|}{$\begin{array}{l}\text { VOLUMEN POR TROZA (m3 s.s.c.) } \\
\text { (trozas de } 3,35 \mathrm{~m} \text { de largo; diámetro menor s.c. }>10 \mathrm{~cm} \text { ) }\end{array}$} & \multirow{2}{*}{$\begin{array}{l}\text { VOLUMEN } \\
\text { ACUM. } \\
\left(\mathrm{m}^{3} \text { s.s.c.) }\right.\end{array}$} \\
\hline & TROZA 1 & TROZA 2 & TROZA 3 & TROZA 4 & TROZA 5 & TROZA 6 & \\
\hline 20 & 0,082 & 0,078 & & & & & 0,160 \\
\hline 25 & 0,152 & 0,114 & 0,108 & 0,065 & & & 0,439 \\
\hline 30 & 0,224 & 0,156 & 0,135 & 0,111 & 0,080 & & 0,706 \\
\hline 35 & 0,298 & 0,205 & 0,172 & 0,154 & 0,125 & & 0,954 \\
\hline 40 & 0,374 & 0,261 & 0,217 & 0,195 & 0,161 & 0,120 & 1,328 \\
\hline 45 & 0,452 & 0,324 & 0,270 & 0,234 & 0,189 & 0,135 & 1,604 \\
\hline 50 & 0,532 & 0,393 & 0,332 & 0,271 & 0,208 & 0,138 & 1,874 \\
\hline
\end{tabular}

\section{REFERENCIAS BIBLIOGRAFICAS}

1. GROSSE H. y KANNEGIESSER U. (1988): Investigación para el manejo de las plantaciones de Pino Oregón y Sequoia sempervirens. (Informe 1988 INFOR - CORFO). 199 pp.

2. HUSCH B.; MILLER, CH. y BEERS, T. (1982): Forest Mensuration. John Wiley \& Sons, Inc. Canadá. $401 \mathrm{pp}$. 